\title{
STEENROD HOMOLOGY AND LOCAL CONNECTEDNESS
}

\author{
JERZY DYDAK
}

\begin{abstract}
Steenrod homology is used to explain results concerning $\mathrm{LC}^{n}$-divisors and one-point compactifications of $\mathrm{LC}^{n}$-spaces. It is shown that the one-point compactification $w X$ of a locally compact metrizable space $X$ is hlc ${ }^{n}$ iff $X$ is hlc ${ }^{n}$ and its Steenrod $k$ th homology is finite generated for $k \leqslant n$.
\end{abstract}

0. Introduction. In $\left[\mathbf{D}_{1}\right]$ the author characterized LC ${ }^{n}$-divisors $(n \geqslant 1)$ as compacta $X$ which are nearly 1-movable and such that pro- $H_{k}(X)$ is stable for $k<n$ and Mittag-Leffler (semistable in today's terminology) for $k=n$. The former condition (near 1-movability) is of homotopy nature and the latter condition is purely homological. It is well known that near 1-movability of $X$ is needed for $M / X$ to be LC $^{1}$ for any $M \in \mathrm{LC}^{n}$ containing $X$ (see [Sh or $\left.\mathbf{D}_{\mathbf{4}}\right]$ ). In this paper it is shown that the homology conditions are needed for $M / X$ to be Steenrod homology locally $n$-connected.

Throughout the paper $\bar{H}_{k}(X)$ denotes the $k$ th Steenrod homology group of $X$ with coefficients in the group of integers (see [M, EH or BM].)

$\bar{H}_{k}^{c}(X)$ denotes the $k$ th Steenrod homology with compact supports.

The author is grateful to Bob Daverman and Gerard Venema for discussions which led to Theorem 3 and its proof.

\section{Locally connected spaces.}

Definition. A locally compact metrizable space $X$ is said to be hlc ${ }^{n}$ if for every compact subset $U$ of $X$ and for every $x \in \operatorname{int} U$ there exists a compact neighborhood $V$ of $x$ in $U$ such that the inclusion induced homomorphism $\tilde{\bar{H}}_{k}(V) \rightarrow \tilde{\bar{H}}_{k}(U)$ is trivial for $k \leqslant n$ (see [BM, p. 150]).

The notion of an hlc ${ }^{n}$-space is dual to the analogous notion of a clc $^{n}$-space which is defined using cohomology rather than homology (see [B, p. 76]). The following lemma is dual to the results in [B, p. 77].

LEMma 1. For a locally compact metrizable space $X$ the following conditions are equivalent:

(a) $X$ is hlc ${ }^{n}$.

(b) For each $x \in X$ and each compact neighborhood $U$ of $x$ there exists a compact neighborhood $V$ of $x$ in $U$ such that $\operatorname{im}\left(\bar{H}_{k}(V) \rightarrow \bar{H}_{k}(U)\right)$ is countable for $k \leqslant n$.

Received by the editors March 11, 1985 and, in revised form, April 24, 1985.

1980 Mathematics Subject Classification. Primary 55N07, 54F35; Secondary 54F43.

Key words and phrases. Steenrod homology, homology locally connected spaces. 
(c) For any two compact subsets $K$ and $L$ of $X$ with $K \subset$ int $L, \operatorname{im}\left(\bar{H}_{k}(U) \rightarrow \bar{H}_{k}(L)\right)$ is finitely generated for $k \leqslant n$.

Proof. (a) $\Rightarrow$ (b) and (c) $\Rightarrow$ (b) are trivial.

(b) $\Rightarrow$ (a) Fix $k \leqslant n$ and choose a decreasing sequence $U_{i+1} \subset U_{i}$ of compact neighborhoods of $x$ in $X$ such that $\{x\}=\bigcap_{i=1}^{\infty} U_{i}$ and $\operatorname{im}\left(\bar{H}_{k}\left(U_{i+1}\right) \rightarrow \bar{H}_{k}\left(U_{i}\right)\right)$ is countable for all $i$.

From the exact sequence $(p \geqslant 0)$

$$
0 \rightarrow \lim ^{1} \tilde{\bar{H}}_{p+1}\left(U_{i}\right) \rightarrow \tilde{\bar{H}}_{p}(x) \rightarrow \operatorname{inv} \lim \tilde{H}_{p}\left(U_{i}\right) \rightarrow 0
$$

(see [EH, p. 270]) we get inv $\lim \tilde{\bar{H}}_{k}\left(U_{i}\right)=\lim ^{1} \tilde{\bar{H}}_{k}\left(U_{i}\right)=0$. A result of Gray [G] says that $\left\{\tilde{H}_{k}\left(U_{i}\right)\right\}$ is semistable, and Proposition 6.1.3 of [DS, p. 77] says that $\left\{\tilde{\bar{H}}_{k}\left(U_{i}\right)\right\}$ is isomorphic as a pro-group to the trivial group. Thus, for each $i$ there exists $m>i$ with $\tilde{\bar{H}}_{k}\left(U_{m}\right) \rightarrow \tilde{\bar{H}}_{k}\left(U_{i}\right)$ being trivial, i.e. $X$ is hlc ${ }^{n}$.

(a) $\Rightarrow$ (c) This is Proposition 6.8 in [BM].

Condition (c) of Lemma 1 gives us the following (see Theorem 6.9 of [BM]).

COROllaRY 1. If $X \in \mathrm{hlc}^{n}$ is compact, then $\bar{H}_{k}(X)$ is finitely generated for $k \leqslant n$.

COROllary 2. Suppose $f: X \rightarrow Y$ is a proper surjection of locally compact metrizable spaces. If $X$ is hlc ${ }^{n}$ and $\tilde{\bar{H}}_{k}\left(f^{-1}(y)\right)=0$ for all $y \in Y$ for $k<n$, then $Y$ is hlc ${ }^{n}$.

Proof. Choose compact subsets $K, L$ of $Y$ with $K \subset$ int $L$. If $K^{\prime}=f^{-1}(K)$, $L^{\prime}=f^{-1}(L)$ then $K^{\prime} \subset$ int $L^{\prime}$ and by Lemma $1 \mathrm{im}\left(\bar{H}_{n}\left(K^{\prime}\right) \rightarrow \bar{H}_{n}\left(L^{\prime}\right)\right)$ is finitely generated. Since $\bar{H}_{n}\left(K^{\prime}\right) \rightarrow \bar{H}_{n}(K)$ is an epimorphism (see [VN or $\mathrm{D}_{3}$ ]) we get that $\operatorname{im}\left(\bar{H}_{n}(K) \rightarrow \bar{H}_{n}(L)\right)$ is finitely generated. By Lemma $1, Y$ is hlc ${ }^{n}$.

THEOREM 1. Let $X$ be a locally compact metrizable space such that $X-\left\{x_{0}\right\}$ is hlc $^{n}$ for some $x_{0} \in X$ and $n \geqslant 0$. If there exists a neighborhood $V$ of $x_{0}$ in $X$ such that the image of the inclusion induced homomorphism $\bar{H}_{k}^{c}(V) \rightarrow \bar{H}_{k}^{c}(X)$ is finitely generated for $k \leqslant n$, then $X$ is hlc ${ }^{n}$.

Proof. Let $U$ be a compact neighborhood of $x_{0}$ in $X$. Choose a compact neighborhood $V$ of $x_{0}$ in $U$ such that $\operatorname{im}\left(\bar{H}_{k}^{c}(V) \rightarrow \bar{H}_{k}^{c}(X)\right)$ is finitely generated. Choose a closed subset $A$ of $X$ such that $X=V \cup A$ and $x_{0} \notin A$. By excision (see $\left[\mathrm{M}\right.$, p. 275]) the inclusion induced homomorphism $\bar{H}_{m}^{c}(A, V \cap A) \rightarrow \bar{H}_{m}^{c}(X, V)$ is an isomorphism for all $m$. From the diagram

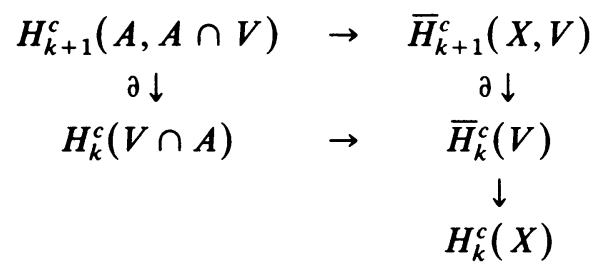

we get that the cokernel of $\bar{H}_{k}^{c}(V \cap A) \rightarrow \bar{H}_{k}^{c}(V)$ is finitely generated for $k \leqslant n$. Indeed, it is an image of the cokernel of $\partial: \bar{H}_{k+1}^{c}(X, V) \rightarrow \bar{H}_{k}^{c}(V)$ which is isomorphic to the image of $\bar{H}_{k}^{c}(V) \rightarrow \bar{H}_{k}^{c}(X)$ known to be finitely generated. Choose $a_{1}, \ldots, a_{m} \in \bar{H}_{n}^{c}(V)$ generating the cokernel of $\bar{H}_{n}^{c}(V \cap A) \rightarrow \bar{H}_{n}^{c}(V)$. Since $X-$ $\left\{x_{0}\right\}$ is hlc , the image of $\bar{H}_{n}^{c}(V \cap A) \rightarrow \bar{H}_{n}^{c}(U)$ is finitely generated (see Lemma 1). 
Choose $b_{1}, \ldots, b_{p} \in \bar{H}_{n}^{c}(V \cap A)$ generating the image of that homomorphism. Then $a_{1}, \ldots, a_{m}, b_{1}, \ldots, b_{p}$ generate the image of $\bar{H}_{n}^{c}(V) \rightarrow \bar{H}_{n}^{c}(U)$. By Lemma $1, X$ is hlc $^{n}$.

COROLlaRY 3. If $X$ is a locally compact metrizable space such that $\bar{H}_{k}^{c}(X)$ is finitely generated for $k \leqslant n$ and $X-\left\{x_{0}\right\}$ is hlc ${ }^{n}$, then $X$ is $\mathrm{hlc}^{n}$.

COROLlaRY 4. Let $X \in \mathrm{hlc}^{n}$ be a noncompact locally compact metrizable space. The one-point compactification $w X$ of $X$ is $\mathrm{hlc}^{n}$ iff $\bar{H}_{k}(X)$ is finitely generated for $k \leqslant n$.

Proof. It follows immediately from Theorem 1 , Corollary 1 and the fact that the groups $\tilde{\bar{H}}_{n}^{c}(w X), \tilde{\bar{H}}_{n}(w X)$, and $\bar{H}_{n}(X)$ are isomorphic (see [M, p. 86]).

Corollary 5. Let $A$ be a compact metrizable space. If $X \in \mathrm{hlc}^{n}$ is a compact metrizable space containing $A$, then the following conditions are equivalent:

(a) $X / A$ is hlc ${ }^{n}$,

(b) $\bar{H}_{k}(A)$ is finitely generated for $k<n$.

Proof. $X / A$ is a one-point compactification of $X-A$. Using Corollary 1 and the exact sequence

$$
\bar{H}_{k}(A) \rightarrow \bar{H}_{k}(X) \rightarrow \bar{H}_{k}(X-A) \rightarrow \bar{H}_{k-1}(A)
$$

(see [M, p. 86]) one establishes the equivalence of conditions (a) and (b).

REMARK. With a little bit more work one can assume in Corollary 5 that $X$ is a locally compact metrizable space.

Corollary 5 and the next result explain the relation between $\left[\mathbf{D}_{1}\right]$ and this paper.

LEMMA 2. For a compact metrizable space $X$ the following conditions are equivalent $(n \geqslant 0)$ :

(a) $\bar{H}_{k}(X)$ is finite generated for $k \leqslant n$,

(b) $\bar{H}_{k}(X)$ is countable for $k \leqslant n$,

(c) pro- $H_{k}(X)$ is stable for $k \leqslant n$ and satisfies the Mittag-Leffler condition for $k=n+1$.

If one of the above conditions is satisfied, then the natural homomorphism $\bar{H}_{k}(X) \rightarrow$ $\check{H}_{k}(X)$ is an isomorphism for $k \leqslant n$.

Proof. Let $X=\operatorname{inv} \lim \left(X_{i}, p_{i}^{i+1}\right)$, where $X_{i}$ is a compact polyhedron for each $i$. Then we have an exact sequence (see [EH, p. 211])

$$
0 \rightarrow \lim ^{1} H_{k+1}\left(X_{i}\right) \rightarrow \bar{H}_{k}(X) \rightarrow \operatorname{inv} \lim H_{k}\left(X_{i}\right) \rightarrow 0
$$

for each $k$. Observe that inv $\lim H_{k}\left(X_{i}\right)$ is simply the Čech $k$ th homology group of $X$. If condition (c) is satisfied, then $\lim ^{1} H_{k}\left(X_{i}\right)=0$ for $k \leqslant n+1$ and $\check{H}_{k}(X)$ is finitely generated for $k \leqslant n$. Therefore $\bar{H}_{k}(X) \rightarrow \check{H}_{k}(X)$ is an isomorphism and condition (a) follows. If condition (b) is satisfied, then $\lim ^{1} H_{k+1}\left(X_{i}\right)$ is countable for $k \leqslant n$ and since each $H_{k+1}\left(X_{i}\right)$ is countable, we have $\lim ^{1} H_{k+1}\left(X_{i}\right)=0$ for $k \leqslant n$ (see [G]). Hence pro- $H_{k}(X)$ is Mittag-Leffler (see [G or DS, p. 78]) for $k \leqslant n+1$ and $\breve{H}_{k}(X)$ is countable for $k \leqslant n$. By Corollary 6.1 .9 of [DS, p. 81], pro- $H_{k}(X)$ is stable for $k \leqslant n$. Thus condition (c) follows from condition (b), and (a) $\Rightarrow$ (b) is trivial. 
LEMMA 3. For a locally compact metrizable space $X$ the following conditions are equivalent $(n \geqslant 1)$ :

(a) $X$ is $\mathrm{LC}^{n}$,

(b) $X$ is hlc ${ }^{n}$ and $X \in \mathrm{LC}^{1}$.

Proof. (a) $\Rightarrow$ (b) Given the compact neighborhoods $U$ and $V$ of $x$ in $X$ with $V \subset$ int $U$ choose a compact neighborhood $W$ of $x$ in $V$ such that $\check{\bar{H}}_{k}(W) \rightarrow \check{\bar{H}}_{k}(V)$ is trivial for $k \leqslant n$ (see [H]). Since pro- $H_{n+1}($ int $U$ ) is Mittag-Leffler (see [D, Theorem 3.3]), the morphism pro- $H_{n+1}(V) \rightarrow$ pro- $H_{n+1}(U)$ factors through a tower of groups which is Mittag-Leffler. Hence $\lim ^{1}\left(\right.$ pro- $\left.H_{n+1}(V)\right) \rightarrow \lim ^{1}\left(\right.$ pro- $\left.H_{n+1}(U)\right)$ is trivial and from the diagram

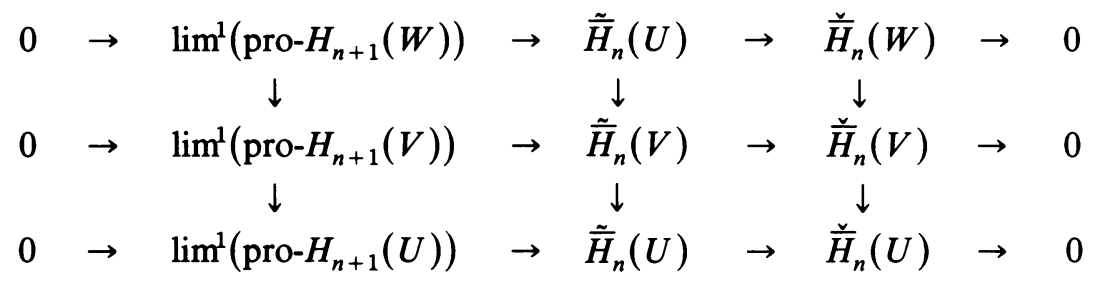

we get that $\tilde{\bar{H}}_{n}(W) \rightarrow \tilde{\bar{H}}_{n}(U)$ is trivial.

(b) $\Rightarrow$ (a) Using a similar diagram as above one gets that for each neighborhood $U$ of $x$ in $X$ there exists a neighborhood $V$ of $x$ in $U$ such that $\check{\bar{H}}_{k}(V) \rightarrow \check{\bar{H}}_{k}(U)$ is trivial for $k \leqslant n$. Now, $X \in \mathrm{LC}^{n}$ follows from the main result of [H].

Using the results of this section one easily proves the main result of $\left[\mathbf{D}_{1}\right]$ :

THEOREM 2. A compact metrizable space $X$ is an $\mathrm{LC}^{n}$-divisor $(n \geqslant 1)$ iff it is an $\mathrm{LC}^{1}$-divisor and pro- $H_{k}(X)$ is stable for $k<n$ and Mittag-Leffler for $k=n$.

As an application of our results we prove the following theorem conjectured by $R$. Daverman and G. Venema.

THEOREM 3. Let $X \in \mathrm{LC}^{n-1}$ be an $n$-dimensional $(n \geqslant 0)$ compactum. If $\check{H}_{n}(X ; \mathbf{Z})$ is finitely generated, then $X \in A N R$.

Proof. From the proof of Lemma 2 one gets that if $\operatorname{dim} Y \leqslant n$, then the natural homomorphism $\bar{H}_{n}(Y) \rightarrow \check{H}_{n}(Y)$ is an isomorphism (the lim ${ }^{1}$ part in the exact sequence vanishes). Hence $\bar{H}_{n}(X)$ is finitely generated and if $A \subset B$ are two compact subsets of $X$, then $\bar{H}_{n+1}(B, A)=0$ and the exact sequence $\bar{H}_{n+1}(B, A) \rightarrow$ $\bar{H}_{n}(A) \rightarrow \bar{H}_{n}(B)$ implies that $\bar{H}_{n}(A) \rightarrow \bar{H}_{n}(B)$ is a monomorphism. Taking $B=X$ we get, by Lemma $1, X \in$ hlc $^{n}$. If $n>1$, then $X \in \mathrm{LC}^{n}$ (by Lemma 3). Since $\operatorname{dim} X=n, X \in \mathrm{ANR}$ (see [Hu, p. 168]). If $n=0$, then $X$ is discrete and $X \in$ ANR.

Suppose $n=1$ and choose a neighborhood $V$ of $x \in X$ such that $\bar{H}_{1}(V) \rightarrow \bar{H}_{1}(X)$ is trivial. Then $\bar{H}_{1}(A)=0$ for all compact subsets $A$ and $V$ and if $f: S^{1} \rightarrow V$ is a map, then $A=f\left(S^{1}\right)$ does not contain any simple closed curve and, therefore, is contractible (see [K, Corollary 7, p. 378]. Thus $X \in \mathrm{LC}^{1}$ and $X \in \mathrm{ANR}$ in view of $\operatorname{dim} X=1$. 


\section{REFERENCES}

[B] G. Bredon, Sheaf theory, McGraw-Hill, New York, 1967.

[BM] A. Borel and J. C. Moore, Homology theory for locally compact spaces, Michigan Math. J. 7 (1960), 137-159.

[D $\mathrm{D}_{1}$ J. Dydak, On LC ${ }^{n}$-divisors, Topology Proc. 3 (1978), 319-333.

$\left[\mathrm{D}_{2}\right]$, Local n-connectivity of quotient spaces and one-point compactifications, Shape Theory and Geom. Top. Proc. (Dubrovnik, 1981), Lecture Notes in Math., vol. 870, Springer-Verlag, Berlin and New York, 1981, pp. 48-72.

$\left[\mathrm{D}_{3}\right] \ldots$, An addendum to the Vietoris-Begle theorem, Topology Appl. (to appear).

$\left[\mathbf{D}_{4}\right]$, Some properties of nearly 1-movable continua, Bull. Acad. Polon. Sci. Sér. Sci. Math. 25 (1977), 685-689.

[DS] J. Dydak and J. Segal, Shape theory: An introduction, Lecture Notes in Math., vol. 688, Springer-Verlag, Berlin and New York, 1978.

[EH] D. A. Edwards and H. M. Hastings, Čech and Steenrod homotopy theories with applications to geometric topology, Lecture Notes in Math., vol. 542, Springer-Verlag, Berlin and New York, 1976.

[G] B. I. Gray, Spaces of the same n-type, for all $n$, Topology 5 (1966), 241-243.

[H] W. Hurewicz, Homotopie, Homologie, und lokaler Zusammenhang, Fund. Math. 25 (1935), 467-485.

[Hu] S.-T. Hu, Theory of retracts, Wayne State Univ. Press, Detroit, Mich., 1965.

[K] K. Kuratowski, Topology, vol. 11, Academic Press, New York, 1968.

[M] W. S. Massey, Homology and cohomology theory, Dekker, New York, 1978.

[Mil] J. Milnor, On axiomatic homology theory, Pacific J. Math. 12 (1962), 337-341.

[Sh] N. Shrinkhande, Homotopy properties of decomposition spaces, Notices Amer. Math. Soc. 22 (1975). Abstract 757-638.

[VN] A. Jn. Volovikov and Nguen Le Anh, On the Vietoris-Begle theorem, Vestnik Moskov. Univ. Ser. I Math. Mekh. (1984), 70-71.

Department of Mathematics, University of Tennessee, Knoxville, Tennessee 37996 